PROJETO DE GRADUAÇÃO EM ENGENHARIA MECÂNICA

\author{
HORTENSE CAUCHARD
}

SIMULAÇÃO DE UM EVAPORADOR OPERANDO COM UMA MISTURA REFRIGERANTE E NANOLUBRIFICANTE

Rio de Janeiro, 03 de Dezembro de 2015 
HORTENSE CAUCHARD

\section{SIMULAÇÃO DE UM EVAPORADOR OPERANDO COM UMA MISTURA REFRIGERANTE E NANOLUBRIFICANTE}

Monografia apresentada ao Departamento de Engenharia Mecânica da Pontifícia Universidade Católica do Rio de Janeiro (PUC-Rio), como requisito parcial para obtenção do título de Engenheiro Mecânico, orientado pelo Prof. José Alberto dos Reis Parise e Coorientado pelo Paul Ortega Sotomayor. 
Agradeço ao orientador e coorientador pela confiança, oportunidade e ajuda.

Ao Ronaldo Galvão pelas sugestões e correções. 


\section{RESUMO}

O trabalho trata da realização de uma simulação de um evaporador ar-ar com superfície externa tipo tubo-aletas operando com uma mistura refrigerante e nanolubrificante. Essa simulação é realizada com o programa EES. O presente modelo envolve a determinação das propriedades do nanofluido e do emprego do método da efetividade.

\section{Palavras-chave:}

Nanofluidos, nanopartícula, evaporador 


\section{ABSTRACT}

This work focuses on the realisation of a simulation of an evaporador air-air type tubesfins using nanofluids. This simulation is made with the EES (Engineering Equation Solver) program. The present study envolve the determination of nanofluids' properties and of the use of the method of effectiveness.

\section{Keywords:}

Nanofluids, nanoparticles, evaporador 


\section{SUMÁRIO}

RESUMO

.iii

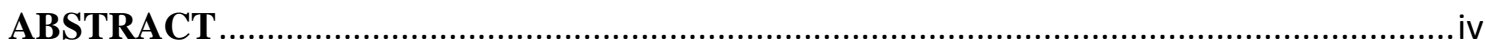

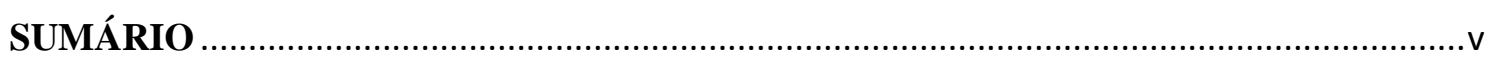

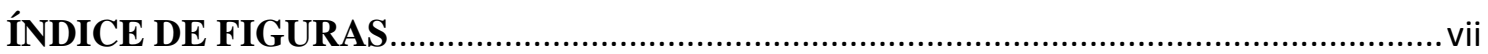

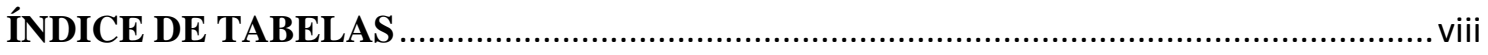

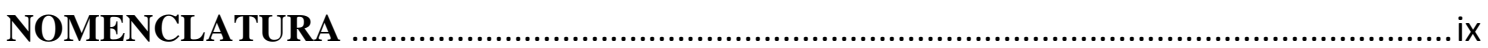

INTRODUÇÃO

I- Sistema de refrigeração e Nanofluidos ................................................................... 2

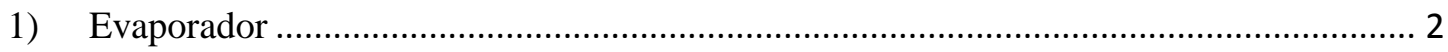

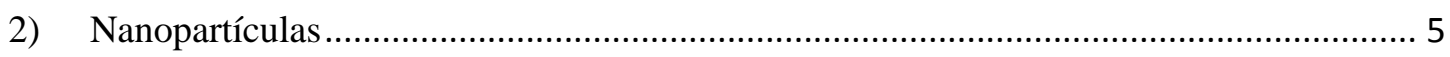

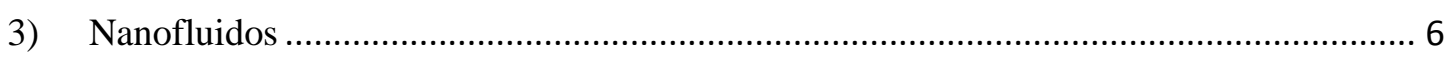

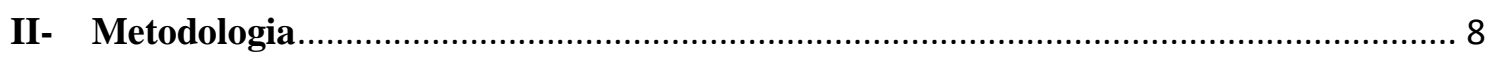

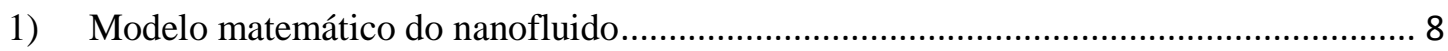

1.1- Propriedades do nanolubrificante ou nano-óleo (óleo e nanopartículas)..................... 8

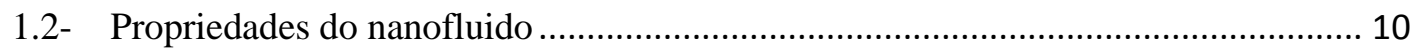

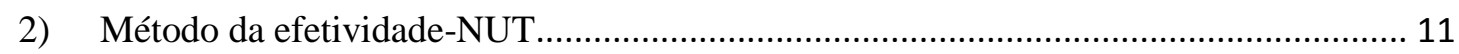

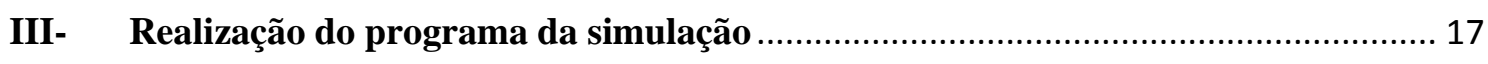

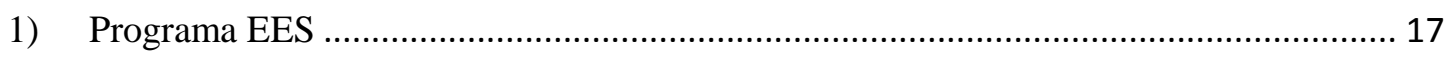

2) Primeira fase: simulação para um evaporador operando com refrigerante .................... 18 
2.2- Desenvolvimento da primeira parte

3) Segunda fase: simulação para um evaporador operando com nanofluidos ..................... 21

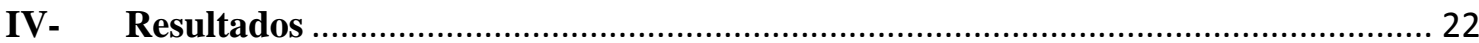

CONCLUSÃO

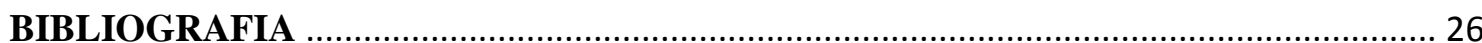




\section{ÍNDICE DE FIGURAS}

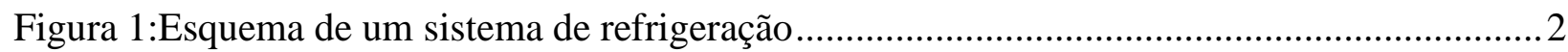

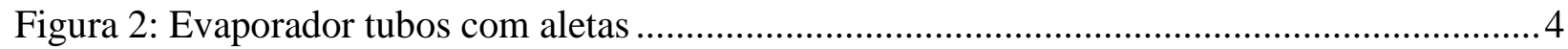

Figura 3: Digrama P-h de um ciclo de refrigeração .............................................................

Figura 4: Nanopartículas vista com microscópio ...............................................................

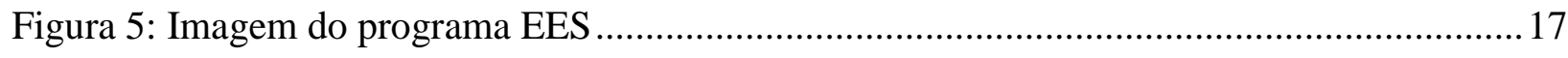

Figura 6: Gráfico da transferência de calor do evaporador global em função do fluido usado ....22

Figura 7: Gráfico da efetividade do evaporador global em função do fluido usado .....................23

Figura 8: Gráfico da temperatura de saida do fluido em função do fluido usado ........................ 23 


\section{ÍNDICE DE TABELAS}

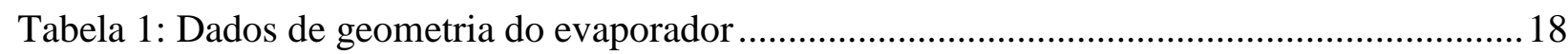

Tabela 2: Dados de entrada do refrigerante ......................................................................... 19

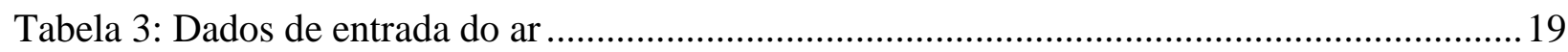




\section{NOMENCLATURA}

\section{$\rho:$ massa específica}

$\varphi_{o l, n p}$ : fração volumétrica das nanopartículas no óleo

$c_{p}:$ calor específica

$k$ : condutividade térmica

$\mu$ : viscosidade dinâmica

$w_{o l, n p}:$ ksjdkjdkqj

$\varepsilon$ : efitividade

$\dot{Q}$ : transferência de calor do evaporador

$\dot{m}$ : vazão mássica

$h$ : enthalpia

$T$ : temperatura

$C$ : capacidade calorifica

$U A$ : condutância

$R$ : resistência térmica

NUT: Número de Unidade de Transferência

$L:$ comprimento

$A$ : área

$\propto$ : coeficiente de troca de calor

$\eta$ : eficiência

Subdivisão:

ol: óleo

$n p$ : nanopartículas 
ol,np: nanolubrificante ou nano-óleo

$r$ : refrigerante

$a:$ ar

$i$ : entrada

$o$ : saída

$e v$ : evaporador

$n f$ : nanofluido

sh: superaquecido

$b f:$ bifásico

fin: aletas 


\section{INTRODUÇÃO}

Busca-se, na refrigeração, melhorar a taxa de transferência de calor do ciclo. Paralelemente, novos refrigerantes têm sido desenvolvidos, em substituição aos atuais que atacam a camada de ozônio e contribuem para o aquecimento global. Esses novos refrigerantes devem possuir as características seguintes: propriedades termodinâmicas favoráveis, baixa toxicidade, não inflamáveis, compatibilidade com materiais, boa estabilidade química, baixo impacto ambiental. Por outro lado, uma nova classe de fluidos com maior transferência de calor tem sido motivo de pesquisa: os nanofluidos. Quando misturadas ao óleo lubrificante, algumas nanopartículas melhorem as condições de lubrificação.

Os objetivos desse projeto são desenvolver um programa de simulação de um evaporador ar-ar operando com uma mistura refrigerante e nanolubrificante e depois avaliar o desempenho desse evaporador utilizando o R134a como fluido base para comparar os resultados utilizando os nanofluidos usando $\mathrm{CuO}$, $\mathrm{TiO} 2$ e $\mathrm{Al} 2 \mathrm{O} 3$ como nanopartículas.

Esse trabalho é dividido em quatro partes. A primeira parte é uma introdução aos sistemas de refrigeração e também aos nanofluidos. A segunda parte trata do modelo matemático usado para realizar o programa. A terceira parte relata os diferentes passos para montar o programa. Enfim, na última parte são apresentados os resultados. 


\section{I- Sistema de refrigeração e Nanofluidos}

Essa parte apresentará uma breve introdução ao sistema de refrigeração, se focalizando no evaporador tubos e aletas. Também será feita a introdução aos nano-fluidos resumindo as principais características e as vantagens de usá-los nos sistemas de refrigeração.

1) Evaporador

A refrigeração é um procedimento permitindo de obter e manter um sistema em uma temperatura inferior à temperatura do meio ambiente (ar condicionado, sistema de refrigeração nos supermercados, pista de gelo...).

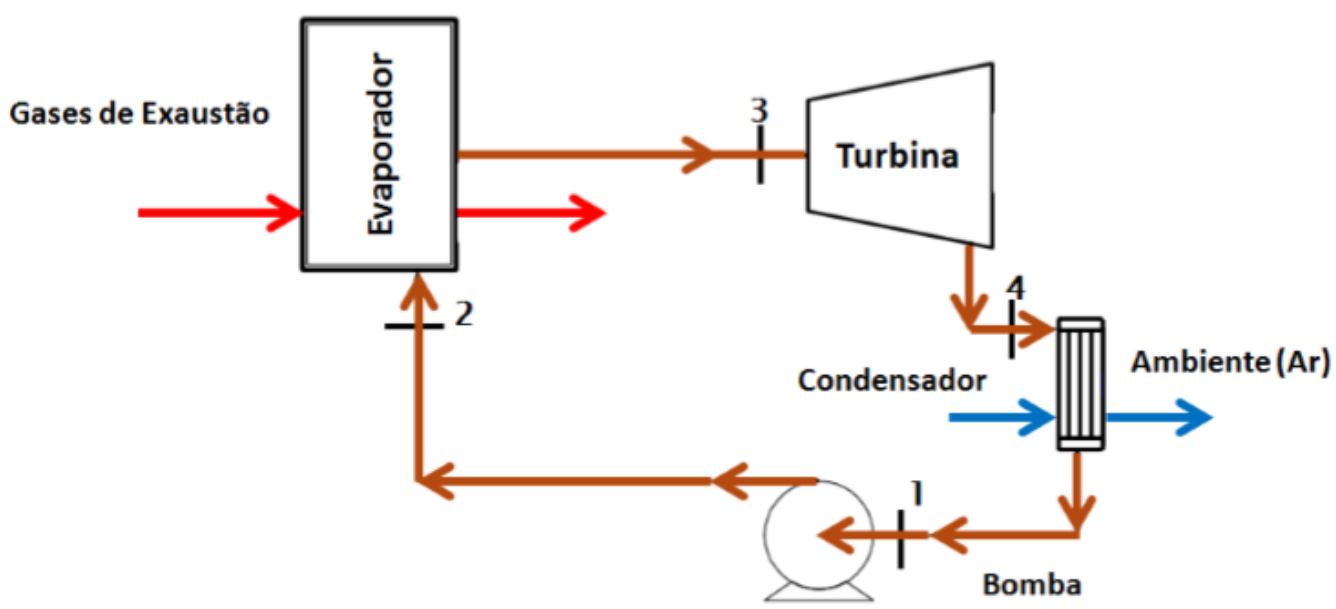

Figura 1:Esquema de um sistema de refrigeração

Ele é constituído de 4 etapas: 
- Evaporação:

Durante essa fase, o evaporador absorva a calor do fluido quente permitindo o refrigerante evaporar.

- Condensação:

Durante a condensação, o condensador rejeita o calor do ciclo no meio ambiente.

- Expansão:

Nessa fase ocorre uma perda de pressão brusca mas controlada de pressão. Essa perde de pressão idealmente isoentâlpica ocorre entre o condensador e o evaporador.

- Compressão:

Durante a compressão, a pressão do fluido refrigerante é elevada. Idealmente, essa compressão é isoentrópica. Mas na realidade tem perda de calor.

Nesse projeto, focamos no evaporador. Como falado antes, um evaporador é um trocador de calor que permite a transferência de energia térmica. A transferência ocorre entre um fluido frio, refrigerante, a baixa temperatura e um fluido quente (ar, água, gás de exaustação...) que possibilita o processo de evaporação.

O tipo de evaporador escolhido para o desenvolvimento desse projeto é o evaporador arar tipo tubos com aletas (mostrado na figura 2). Esse tipo de evaporador é constituído de tubos e de placas finas fixadas entre eles. As aletas melhoram a eficiência da transferência de calor porque eles aumentam a área global de troca de calor. 


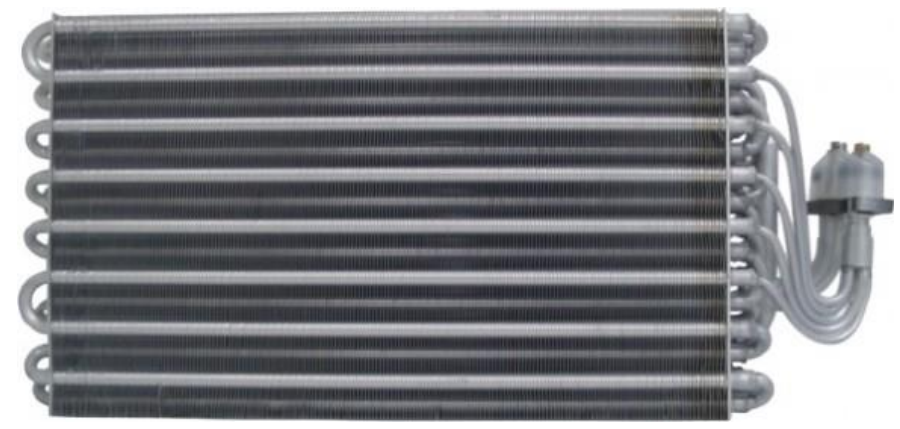

Figura 2: Evaporador tubos com aletas

Como esses evaporadores têm uma área maior, eles podem ser mais compactos sem prejudicar a capacidade de absorção de calor. O material usado nas aletas deve ser um bom condutor de calor, por exemplo alumínio ou cobre.

Quanto menor for a temperatura, mais espaçadas estarão as aletas. Isso é necessário porque o gelo bloqueia com facilidade a circulação do ar no evaporador quando elas estão muito próximas umas das outras. Além disso, os evaporadores de ar natural usam um espaço maior entre as aletas do que um de ar forçado.

Esse evaporador foi escolhido porque ele é o mais utilizado nas máquinas que usamos no dia a dia por exemplo nas geladeiras ou nos supermercados.

O evaporador pode ser dividido em duas partes: uma parte bifásica e uma parte de superaquecimento. Essa divisão é necessária porque as propriedades são diferentes na parte bifásica onde tem uma mistura vapor e liquido das propriedades na parte do superaquecimento. 


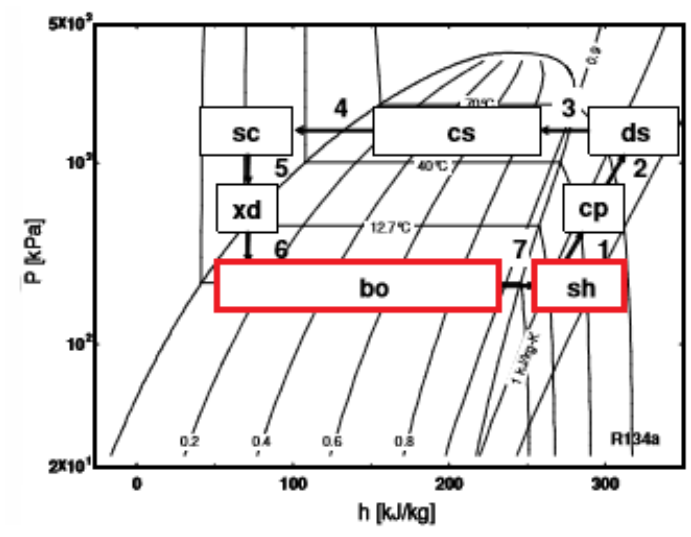

Figura 3: Digrama P-h de um ciclo de refrigeração

No diagrama, as partes Bo (boiler) e Sh (super heat) representam as duas partes (parte bifásica e superaquecimento) do evaporador. Para simplificar, é considerado a fase bifásica (Bo) como uma parte só.

2) Nanopartículas

Uma nanopartícula é um corpo tendo uma dimensão da ordem de 100nm ou menor. Elas têm características (eletrônicas, ópticas, magnéticas, mecânicas, reatividade química) diferentes do material de origem. De fato, as propriedades físicas, químicas e mecânicas são diferentes das propriedades do material de origem.

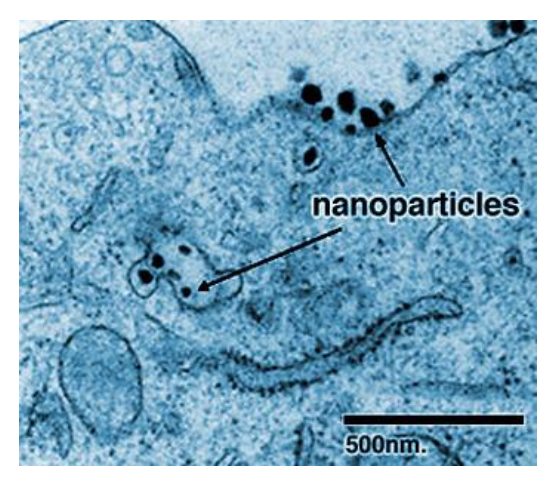

Figura 4: Nanopartículas vista com microscópio 
A maioria é fabricado sob a forma de pós secos mas em alguns segundos eles se aglomerarão rapidamente através de um mecanismo de agregação, o que pode prejudicar a aplicação do nanomaterial. Para ser conservadas separadamente, elas devem preparadas e conservadas em um líquido que permite ter uma repulsão suficiente entre as partículas para não ter agregação.

\section{3) Nanofluidos}

O objetivo principal buscado na refrigeração é de melhorar a taxa de transferência de calor do ciclo. Uma das maneiras de melhorá-la é buscar novos refrigerantes dos que já estão usados. Esses novos refrigerantes devem possuir as características seguinte:

- Propriedades termodinâmicas favoráveis

- Baixa toxicidade

- Não inflamável

- Compatibilidade com materiais

- Boa estabilidade química

- Baixo impacto ambiental

Uma nova classe de fluidos com maior transferência de calor tem sido motivo de pesquisa: os nanofluidos. Os nanofluidos são fluidos térmicos obtidos da suspensão de partículas de tamanho manométrico (comprimento entre $1 \mathrm{~nm}$ e $100 \mathrm{~nm}$ ) em fluidos convencionais de transferência de calor, usualmente líquidos. $\mathrm{O}$ fato deles ter uma transferência de calor maior pode ser por causa da maior ordem de grandeza da condutividade térmica dos sólidos. 
Adicionar nanopartículas aos fluidos refrigerantes e óleos lubrificantes permitem significados aumentos nas propriedades termofísicas dos fluidos. Nesse projeto, consideramos como fluido de base uma mistura de um fluido refrigerante com óleo lubrificante.

As nanopartículas podem também melhorar a solubilidade do óleo mineral com o refrigerante. É o que observou Elcock, em 2007, com as nanopartículas de dióxido de titânio ( $\left.\mathrm{TiO}_{2}\right)$ no refrigerante hidrofluorocarboneto (HFC). Ele observou também que essas nanopartículas melhoram o desempenho dos sistemas de refrigeração que utilizam uma mistura de R314a (refrigerante) e de óleo mineral.

A adição de nanopartículas no refrigerante permite diminuir a massa específica e aumentar a viscosidade, o calor específico e a condutividade térmica. $\mathrm{O}$ aumento da condutividade térmica permite ter um ganho na transferência de calor, o que é uma vantagem. Ao contrário, o aumento da viscosidade não é favorável nos sistemas de refrigeração porque aumenta a queda de pressão. 


\section{II- Metodologia}

Nessa parte será desenvolvido o modelo matemático, baseado na literatura, para o cálculo das propriedades termofísicas seguintes: condutividade térmica, viscosidade, calor específico e massa específica da mistura refrigerante e lubrificante ou nano-óleo (óleo e nanopartículas). Será desenvolvido também o método usado para montar o programa de simulação do evaporador.

1) Modelo matemático do nanofluido

1.1- Propriedades do nanolubrificante ou nano-óleo (óleo e nanopartículas)

Um nanolubrificante ou nano-óleo é a mistura do óleo com nanopartículas. As correlações apresentadas abaixo foram encontradas na literatura. O subscrito $(n p)$ é utilizado para fazer referência às nanopartículas e o subscrito $(o l)$ para fazer referência ao óleo.

- Massa específica $\left(\rho_{o l, n p}\right)$ :

A massa específica é calculada em função da fração volumétrica das nanopartículas no óleo $\left(\varphi_{o l, n p}\right)$ e das massas específicas da nanopartícula $\left(\rho_{n p}\right)$ e do óleo $\left(\rho_{o l}\right)$.

$$
\rho_{o l, n p}=\left(1-\varphi_{o l, n p}\right) * \rho_{o l}+\varphi_{o l, n p} * \rho_{n p}
$$


- Calor específico $\left(c_{p, o l, n p}\right)$ :

O calor específico é calculado em função da massa específica do nano-óleo $\left(\rho_{o l, n p}\right)$, o calor específico do óleo $\left(c_{p, o l}\right)$, o calor específico da nanopartícula $\left(c_{p, n p}\right)$ e da fração volumétrica das nanopartículas no óleo $\left(\varphi_{o l, n p}\right)$.

$$
c_{p, o l, n p}=\frac{\left(1-\varphi_{o l, n p}\right) * \rho_{o l} * c_{p, o l}+\varphi_{o l, n p} * \rho_{n p} * c_{p, n p}}{\rho_{o l, n p}}
$$

- Condutividade térmica $\left(k_{o l, n p}\right)$ :

A condutividade térmica é calculada em função da condutividade térmica do óleo $\left(k_{o l}\right)$, da condutividade térmica da nanopartícula $\left(k_{n p}\right)$ e da fração volumétrica das nanopartículas no óleo $\left(\varphi_{o l, n p}\right)$.

$$
k_{o l, n p}=k_{o l} \frac{k_{n p}+2 k_{o l}-2 \varphi_{o l, n p} *\left(k_{o l}-k_{n p}\right)}{k_{n p}+2 k_{o l}+\varphi_{o l, n p} *\left(k_{o l}-k_{n p}\right)}
$$

- Viscosidade dinâmica $\left(\mu_{o l, n p}\right)$ :

A viscosidade dinâmica é calculada em função da viscosidade dinâmica do óleo $\left(\mu_{o l}\right)$ e da fração volumétrica das nanopartículas no óleo $\left(\varphi_{o l, n p}\right)$. 


$$
\mu_{o l, n p}=\mu_{o l} \frac{1}{\left(1-\varphi_{o l, n p}\right)^{2.5}}
$$

\section{2- $\quad$ Propriedades do nanofluido}

O nanofluido corresponde a mistura do fluido refrigerante e do nano-óleo (óleo e nanopartículas). O subscrito $(n f)$ é utilizado para fazer referência ao nanofluido e o subscrito $(r)$ para fazer referência ao refrigerante.

- Massa específica $\left(\rho_{n f}\right)$ :

A massa específica é calculada em função da concentração do nano-óleo no nanofluido $\left(w_{o l, n p}\right)$ e das massas específicas da nano-oleo $\left(\rho_{o l, n p}\right)$ e do refrigerante $\left(\rho_{r}\right)$. A faixa de temperatura para o uso desta equação é de $-20^{\circ} \mathrm{C}$ até $80^{\circ} \mathrm{C}$.

$$
\rho_{n f}=\left[\frac{w_{o l, n p}}{\rho_{o l, n p}}+\left(\frac{1-w_{o l, n p}}{\rho_{r}}\right)\right]^{-1}
$$

- Calor específico $\left(c_{p, n f}\right)$ :

O calor específico é calculado em função da massa específica do nano-óleo $\left(\rho_{o l, n p}\right)$, a massa específica do refrigerante $\left(\rho_{r}\right)$, a massa específica do nanofluido $\left(\rho_{n f}\right)$, o calor específico do refrigerante $\left(c_{p, r}\right)$, o calor específico do nano-óleo $\left(c_{p, o l, n p}\right)$ e da concentração do nano-óleo no nanofluido $\left(w_{o l, n p}\right)$. 


$$
c_{p, n f}=\frac{\left(1-w_{o l, n p}\right) * \rho_{r} * c_{p, r}+w_{o l, n p} * \rho_{o l, n p} * c_{p, o l, n p}}{\rho_{n f}}
$$

- Condutividade térmica $\left(k_{n f}\right)$ :

A condutividade térmica é calculada em função da condutividade térmica do nano-óleo $\left(k_{o l, n p}\right)$, da condutividade térmica do refrigerante $\left(k_{r}\right)$ e da concentração do nano-óleo no nanofluido $\left(w_{o l, n p}\right)$

$$
k_{n f}=k_{r} *\left(1-w_{o l, n p}\right)+\left(k_{o l, n p} * w_{o l, n p}\right)-\left(0.72 w_{o l, n p} *\left(1-w_{o l, n p}\right)\left(k_{o l, n p}-k_{r}\right)\right.
$$

- Viscosidade dinâmica $\left(\mu_{n f}\right)$ :

A viscosidade dinâmica é calculada em função da viscosidade dinâmica do nano-óleo $\left(\mu_{o l, n p}\right)$, da viscosidade dinâmica do refrigerante $\left(\mu_{r}\right)$ e da concentração do nano-óleo no nanofluido $\left(w_{o l, n p}\right)$.

$$
\mu_{n f}=e^{\left[w_{o l, n p} * \ln \left(\mu_{o l, n p}\right)+\left(1-w_{o l, n p}\right) * \ln \left(\mu_{r}\right)\right]}
$$

\section{2) Método da efetividade-NUT}

Existe dois métodos para realizar a simulação do trocador: 
- O método da efetividade-NUT

- O método da média logarítmica das diferenças de temperaturas

A escolha do método da efetividade foi feito porque o tempo do cálculo é menor do que o tempo do cálculo do método da média logarítmica das diferenças de temperaturas. De fato, o método das temperaturas logarítmicas tem muitas iterações que aumenta o tempo de cálculo. Além disso é possível determinar a taxa de transferência de calor só com as temperaturas de entrada dos fluidos.

As equações do método da efetividade para um evaporador são as seguintes:

- Efetividade do trocador de calor $(\varepsilon)$ :

A efetividade pode ser determinada com a taxa de transferência de calor real $(q)$ e a taxa de transferência de calor máxima $\left(q_{\max }\right)$.

$$
\varepsilon=\frac{q}{q_{\max }}
$$

- Os balanços de energia:

O balanço de energia do lado refrigerante é a igualdade entre a transferência de calor do evaporador $\left(\dot{Q}_{e v}\right)$ e uma função da entalpia de entrada do refrigerante $\left(h_{r, i}\right)$, da entalpia de saída do refrigerante $\left(h_{r, o}\right)$ e da vazão mássica do refrigerante $\left(\dot{m}_{r}\right)$.

$$
\dot{Q}_{e v}=\dot{m}_{r} *\left(h_{r, o}-h_{r, i}\right)
$$


O balanço de energia do lado do ar é a igualdade entre a transferência de calor do evaporador $\left(\dot{Q}_{e v}\right)$ e uma função da temperatura de entrada do ar $\left(T_{a, i}\right)$, da temperatura de saída do $\operatorname{ar}\left(T_{a, o}\right)$, da vazão mássica do ar $\left(\dot{m}_{a}\right)$ e da calor específica do ar $\left(c_{p, a}\right)$.

$$
\dot{Q}_{e v}=\dot{m}_{a} * c_{p, a} *\left(T_{a, i}-T_{a, o}\right)
$$

- A equação da efetividade:

Essa equação igualiza a transferência de calor do evaporador $\left(\dot{Q}_{e v}\right)$ com uma função da efetividade $(\varepsilon)$, com a taxa de capacidade calorífica mínima $\left(C_{\min }\right)$, a temperatura de entrada do $\operatorname{ar}\left(T_{a, i}\right)$ e a temperatura de entrada do refrigerante $\left(T_{r, i}\right)$.

$$
\dot{Q}_{e v}=\varepsilon * C_{\min } *\left(T_{a, i}-T_{r, i}\right)
$$

Sabendo que $C_{\min }$ corresponde à taxa de capacidade calorífica mínima:

$$
C_{\min }=\operatorname{MIN}\left(C_{r} ; C_{a}\right)
$$

Com:

$$
\begin{aligned}
& C_{r}=\dot{m}_{r} * c_{p, r} \\
& C_{a}=\dot{m}_{a} * c_{p, a}
\end{aligned}
$$


- A equação da condutância $(U A)$ :

A condutância é calculada em função das resistências térmicas: $R_{r}$ (resistência térmica do refrigerante), $R_{w}$ (resistência térmica do tubo), $R_{a}$ (resistência térmica do ar) $\mathrm{e}$ consequentemente da geometria do evaporador.

$$
\frac{1}{U A}=R_{r}+R_{w}+R_{a}
$$

As resistências térmicas são, de fato, calculadas com a geometria do evaporador.

$$
R_{r}=\frac{1}{\propto_{r} * \pi * D_{\text {int }} * L_{\text {tubo }}}
$$

Sabendo que $\propto_{r}$ é o coeficiente de troca de calor do refrigerante, $D_{\text {int }}$ o diâmetro interno dos tubos e $L_{\text {tubo }}$ o comprimento dos tubos.

$$
R_{a}=\frac{1}{\propto_{a} * A_{a} * \eta_{0}}
$$

Sabendo que $\propto_{a}$ é o coeficiente de troca de calor do ar e: 


$$
\begin{aligned}
& A_{a}=A_{a, \text { fin }}+A_{a, \text { outfin }} \\
& \eta_{0}=1-\frac{A_{a, \text { fin }}}{A_{a}} *\left(1-\eta_{\text {fin }}\right)
\end{aligned}
$$

Onde $A_{a, \text { fin }}$ é a área das aletas, $A_{a, \text { outfin }}$ a área da base sem aletas e $\eta_{\text {fin }}$ é a eficiência das aletas.

A última resistência térmica $R_{w}$ é considerada como desprezível nesse caso porque a espessura dos tubos é muito fina então a resistência é muito baixa e pode não ser levado em conta.

- A equação do Número de Unidades de Transferência (NUT):

$$
N U T=\frac{U A}{C_{\min }}
$$

Quanto maior o NUT é, maior é o trocador.

- A equação da efetividade em função do NUT:

A efetividade é uma função do NUT e da relação de capacidades caloríficas.

$$
\varepsilon=f(N U T, c)
$$

\section{Com}

$$
c=\frac{C_{\min }}{C_{\max }}
$$




$$
C_{\max }=\operatorname{MAX}\left(C_{r} ; C_{a}\right)
$$

Então para valores baixos de NUT, o valor da efetividade vai estar baixo também. Num trocador ideal NUT tem um valor infinito (porque A tem um valor infinito) para $q \max =C \min * \Delta \operatorname{Tmax}$.

No caso de um evaporador precisamos tomar em conta as duas fases: fase bifásica e fase de super aquecimento, como explicado anteriormente. Assim precisamos dividir o trabalho em duas partes (parte bifásica e parte do super aquecimento) e aplicar o método da efetividade nas duas partes. Para obter a efetividade total do evaporador basta adicionar a efetividade achada no cálculo da fase bifásica e a efetividade achada no cálculo da parte do super aquecimento.

A formula da efetividade em função do NUT na fase bifásica é:

$$
\varepsilon=1-e^{-N T U}
$$

A formula da efetividade em função do NUT na fase do super aquecimento é:

$$
\varepsilon=\frac{1-e^{-N T U *\left(1-C_{r}\right)}}{1-C_{r} * e^{-N T U *\left(1-C_{r}\right)}}
$$

Com

$$
C_{r}=\frac{C_{\min }}{C_{\max }}
$$




\section{III- Realização do programa da simulação}

Nessa seção, será apresentado o programa EES e também as diferentes etapas do desenvolvimento do programa da simulação.

\section{1) Programa EES}

O programa usado para montar a simulação se chama programa EES (Engineering Equation Solver). Esse programa permite resolver numericamente equações não-linear e diferencial. Ele pode ser usado para fazer otimizações, regressões linear e não-linear, converter unidades... Uma das vantagens do programa é a sua precisão termodinâmica e seu banco de dados para propriedades de centenas de fluidos permitindo a sua capacidade a resolver as equações termodinâmicas.

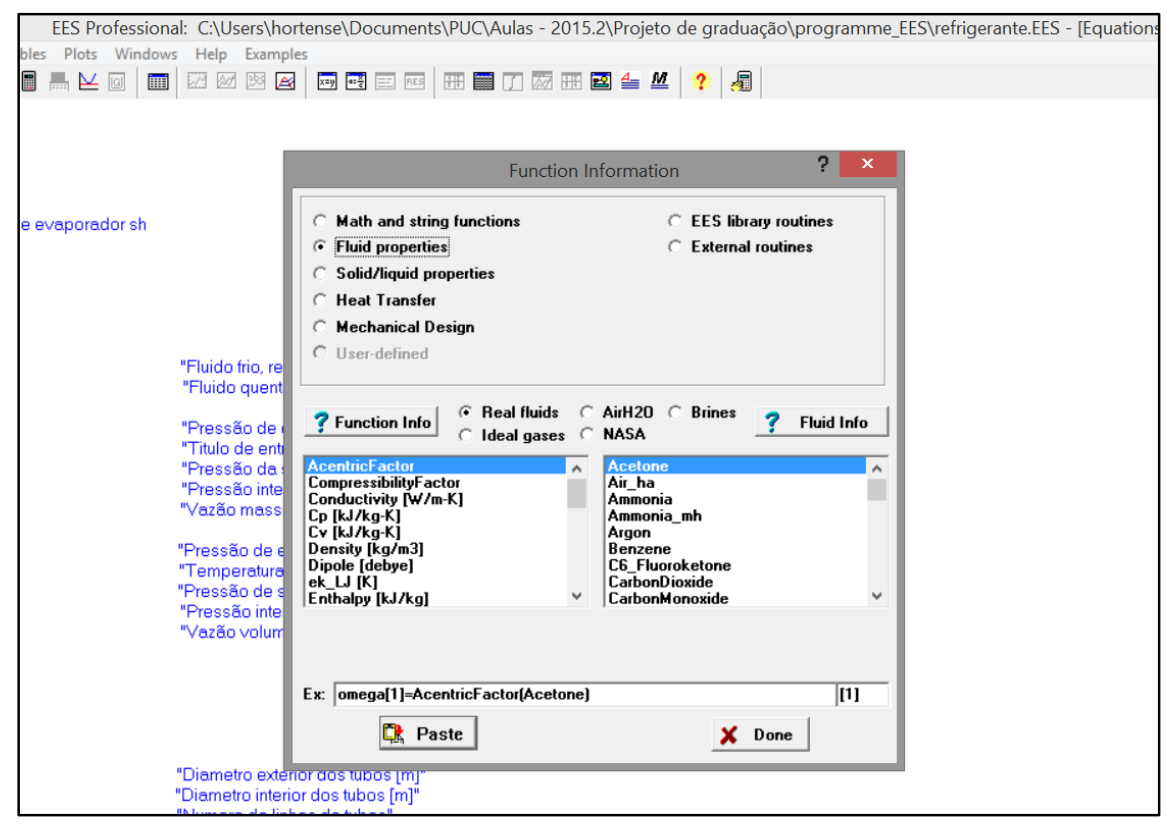

Figura 5: Imagem do programa EES 
As equações no programa EES podem estar montadas em qualquer ordem e a resolução das equações é muito rápida. Para que o programa resolva as equações, precisa de ter o mesmo número de variáveis e de equações.

\section{2) Primeira fase: simulação para um evaporador operando com refrigerante}

A realização do programa foi feita em duas partes. A primeira parte é a criação do modelo de simulação para o sistema de refrigeração tradicional usando um refrigerante normal.

\section{1- Dados de entrada}

Os dados de entrada são dados experimentais usados sobre um evaporador ar-ar tipo tubos e aletas trabalhando com um refrigerante R314a.

Os dados são listados nas tabelas abaixas:

\begin{tabular}{|c|c|c|}
\hline \multicolumn{3}{|c|}{ Geometria do evaporador } \\
\hline L_tubo & $\mathrm{mm}$ & 290 \\
\hline Dint & $\mathrm{mm}$ & 6,8 \\
\hline Dext & $\mathrm{mm}$ & 7,4 \\
\hline eta_fin & - & 0,8 \\
\hline p_fin & $\mathrm{mm}$ & 18,5 \\
\hline N_t_col & - & 3 \\
\hline N_t_lin & - & 15 \\
\hline t_fin & $\mathrm{mm}$ & 0,11 \\
\hline H & $\mathrm{m}$ & 0,3885 \\
\hline W & $\mathrm{m}$ & 0,0777 \\
\hline
\end{tabular}

Tabela 1: Dados de geometria do evaporador 


\begin{tabular}{|c|c|c|}
\hline \multicolumn{3}{|c|}{ dados do refrigerante } \\
\hline P_r_1 & $\mathrm{kPa}$ & 690 \\
\hline X_r_1 & - & 0,156 \\
\hline T_r_1 & ${ }^{\circ} \mathrm{C}$ & 26.0 \\
\hline m_dot_r & $\mathrm{kg} / \mathrm{s}$ & 0,0172 \\
\hline P_r_2 & $\mathrm{kPa}$ & 660 \\
\hline P_r_i & $\mathrm{kPa}$ & 660 \\
\hline
\end{tabular}

Tabela 2: Dados de entrada do refrigerante

\begin{tabular}{|c|c|c|}
\hline \multicolumn{3}{|c|}{ dados do ar } \\
\hline P_a_1 & $\mathrm{kPa}$ & 92 \\
\hline T_a_1 & ${ }^{\circ} \mathrm{C}$ & 30 \\
\hline m_dot_a & $\mathrm{kg} / \mathrm{s}$ & 0,974 \\
\hline P_a_2 & $\mathrm{kPa}$ & 92 \\
\hline P_a_i & $\mathrm{kPa}$ & 92 \\
\hline
\end{tabular}

Tabela 3: Dados de entrada do ar

\section{2- Desenvolvimento da primeira parte}

Na primeira parte o método da efetividade é aplicado ao sistema de refrigeração usando um fluido refrigerante normal, o R314a. Como falado antes, o programa é dividido em duas partes; parte bifásica e parte do superaquecimento. Então, precisa dividir o programa em duas partes também. Assim o método da efetividade vai ser aplicada na parte bifásica para obter $\dot{Q}_{e v, b f}$, a transferência de calor do lado bifásico. Da mesma maneira será calculada a transferência de calor do lado do superaquecimento $\dot{Q}_{e v, s h}$.

Usando a soma dessas duas transferências e os balanços de energia global do evaporador, podemos encontrar a transferência do evaporador total $\dot{Q}_{e v, t o t}$ e a efetividade total $\varepsilon_{t o t}$. 
Uma vez que as equações do método da efetividade foram entradas, é importante verificar que o número de equações corresponde ao número de variáveis para rodar o programa. Nesse programa, faltam duas equações para fechar os cálculos.

Para resolver esse problema, usa-se uma função objetivo à minimizar. Para influenciar o cálculo inteiro a função objetivo precisa ser global. A função objetivo é:

$$
\begin{aligned}
& f_{o b j}=\left|\dot{Q}_{e v, t o t}-\dot{Q}_{e v}\right| \\
& \text { Com } \\
& \dot{Q}_{e v, t o t}=\dot{Q}_{e v, s h}+\dot{Q}_{e v, b f} \\
& \dot{Q}_{e v}=\varepsilon_{t o t} * C_{\text {min }} *\left(T_{a, i}-T_{r, i}\right)
\end{aligned}
$$

A equação (29) corresponde ao cálculo da transferência de calor do evaporador total somando as da parte bifásica e da parte do superaquecimento. A equação (30) corresponde ao cálculo da transferência do evaporador total com o método da efetividade. O $\dot{Q}_{e v}$ e o $\dot{Q}_{e v, t o t}$ deveriam estar iguais então podemos minimizar a função objetivo que deveria estar igual a 0 . Para realizar isso, o programa EES tem uma função minimização onde precisa escolher a função a minimizar (função objetivo) e duas variáveis que não conhecemos para obter os valores permitindo a melhor minimização da função. Assim podemos obter os resultados da simulação do evaporador ar-ar tipo tubos e aletas operando com o refrigerante R134a. 
3) Segunda fase: simulação para um evaporador operando com nanofluidos

Uma vez que a simulação operando com um refrigerante normal funciona podemos aplicá-la aos nanofluidos. Para isso precisamos adicionar uma sub-rotina contendo os cálculos das propriedades dos nanofluidos a partir do tipo de nanopartículas, do fluido, da fração mássica das nanopartículas no óleo e da fração mássica do nano-óleo no refrigerante.

Depois da adição da sub-rotina, a simulação está pronta para usar. 


\section{IV- Resultados}

Nessa parte serão apresentados os resultados obtidos com a simulação.

O programa foi usado com um refrigerante normal R314a, com três nanofluidos usando nanopartículas de alumina (Al2O3), de oxido de cobre $(\mathrm{CuO})$ e de titânia (TiO2) com as mesmas fações mássicas: $w_{o l, n p}=0.1$ (fração mássica do nano-óleo no nanofluido) e $w_{n p}=0.1$ (fração mássica das nanopartículas no nano-óleo).

Os resultados obtidos são reportados nos gráficos abaixos:

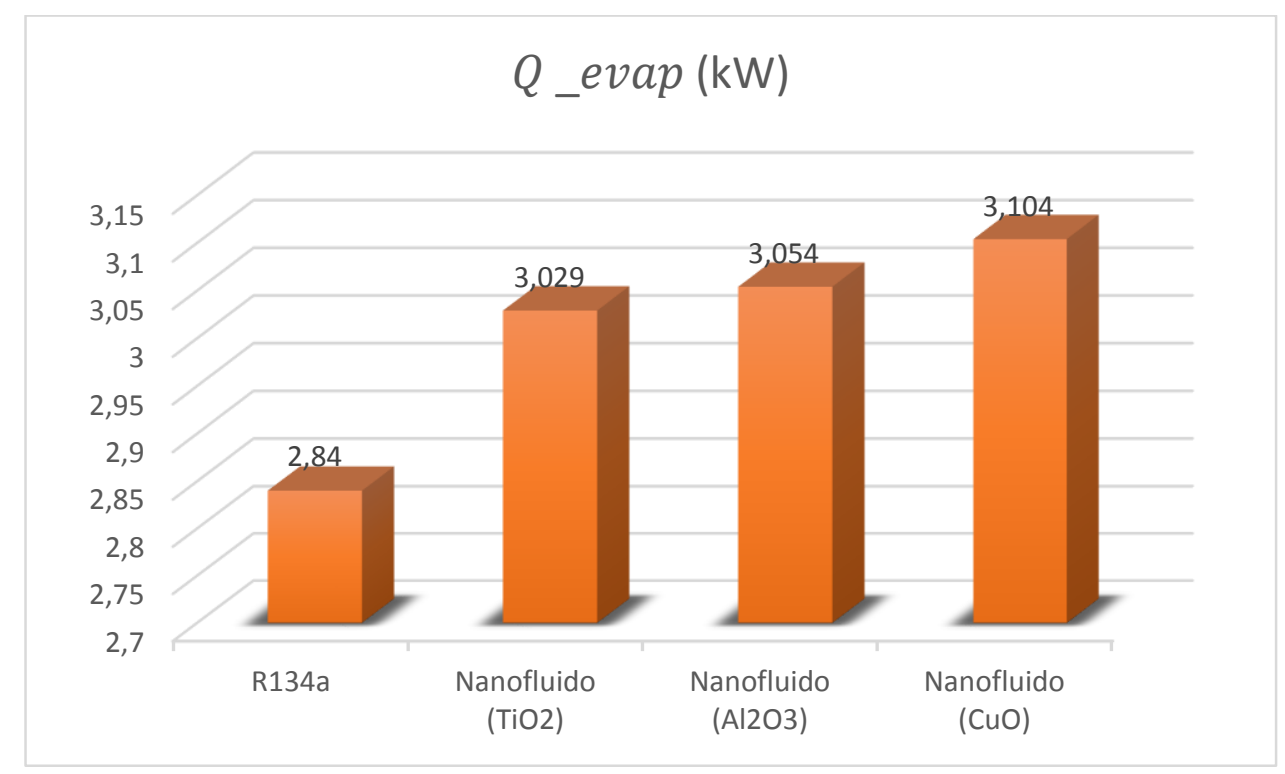

Figura 6: Gráfico da transferência de calor do evaporador global em função do fluido usado 


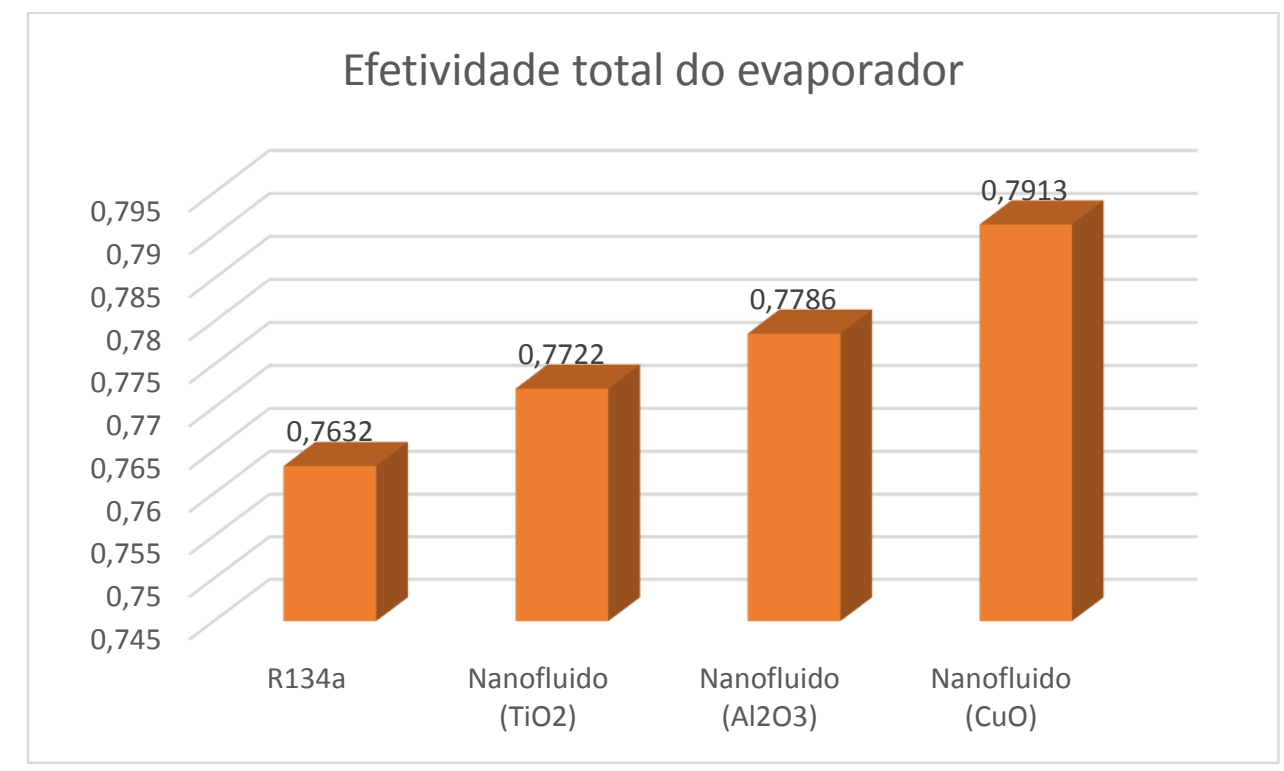

Figura 7: Gráfico da efetividade do evaporador global em função do fluido usado

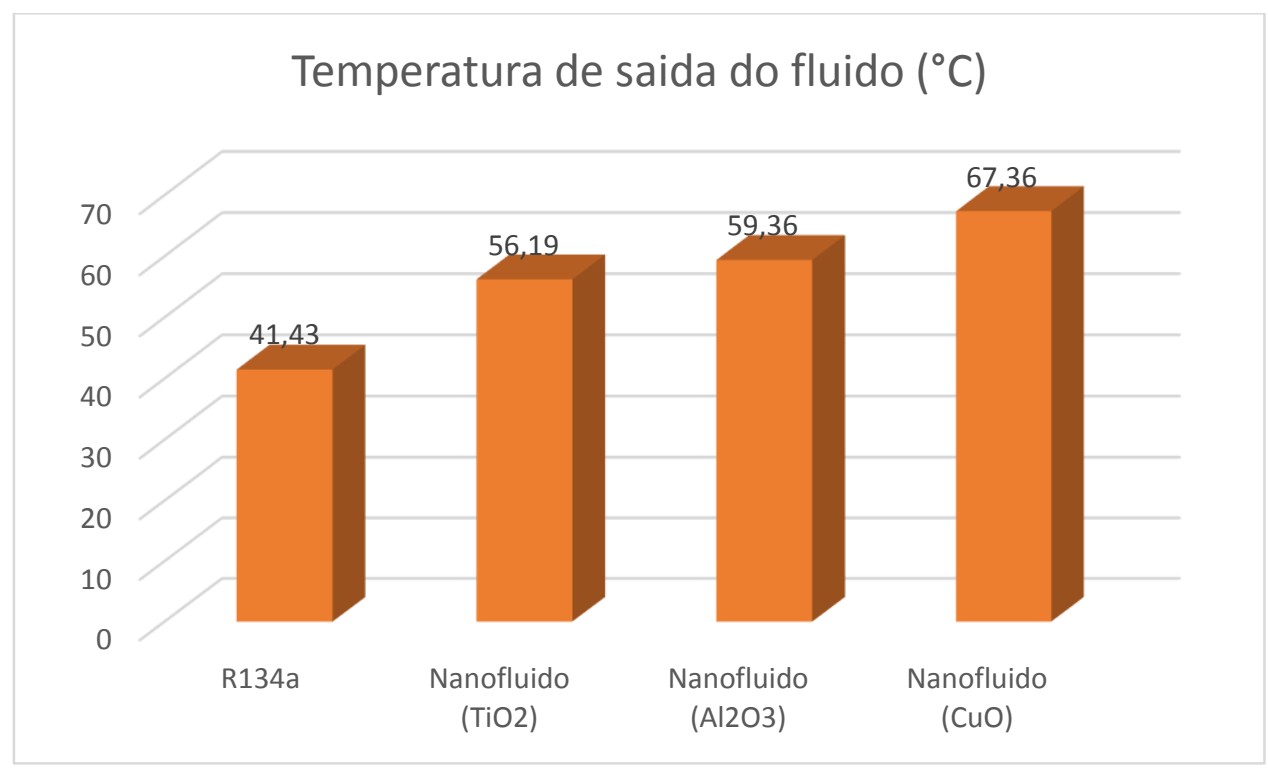

Figura 8: Gráfico da temperatura de saida do fluido em função do fluido usado

Com esses resultados, podemos constatar que o desempenho do evaporador é maior quando usado com nanofluidos. A troca de calor, a efetividade e a temperatura de saída do fluido são maiores (e melhores) ao usar dos nanofluidos. Podemos também relatar que cada nanopartícula 
tem propriedades diferentes então é normal de obter resultados diferentes usando um nanofluido com oxido de cobre ou com titânia por exemplo. 


\section{CONCLUSÃo}

Foi possível realizar a simulação a partir de dados experimentais de um evaporador operando com refrigerante e adaptando-a aos nanofluidos.

Essa simulação permite entender os resultados que serão obtidos usando nanofluidos no evaporador.

Os resultados encontrados concordam com a teoria e mostram que os nanofluidos podem ser o futuro do fluido refrigerante nos sistemas de refrigeração. 


\section{BIBLIOGRAFIA}

1- http://www.ppgem.ct.utfpr.edu.br/lacit/publicacoes/projetos_finais/PF\%202004\%20\%20Diego-Rafael-Anzolin.PDF

2- http://sereva.es/index.php/fr/produits/evaporateurs-statiques/evaporateur-ma

3- http://lqes.iqm.unicamp.br/canal_cientifico/lqes_responde/lqes_responde_nanoparticula.h tml

4- J. Parise, J. Neto, P. Ortega, “Determinação das propriedades termofisicas”, Mercofrio 2014 - 9 Congresso Internacional de ar condicionado, refrigeração, aquecimento e ventilação, Agosto 2014, p1-9

5- Rohsenow, W. M., J. P. Hartnett, and Y. I. Cho, Y. I., Handbook of Heat Transfer, 3rd edition, McGraw-Hill, (1998) 